referred to or published in new papers in its own journals, and offers its facilities to other societies publishing original research. It should be emphasized that deposition of spectra is voluntary on the part of the author, with the object of the spectra being generally available in a form larger than can be printed; it is not a condition of acceptance of papers for publication. All spectra deposited with the Society must be drawn in indian ink, with all other lettering in pencil, on forms obtainable from the General Secretary, Chemical Society, Burlington House, Piccadilly, London, W.1, price $6 s$. per pad of fifty sheets. Photocopies of speetra thus deposited are made available to the public on request to the general secretary of the Society; applications must quote the C.S. number of the spectra desired and must be accompanied by a remittance of $3 s$. per copy per spectrum (post-paid), special rates being available for a large number ordered at one time. Fifty spectra which are already available will be listed in Proceedings of the Chemical Society of December 1953 ; further lists will be published quarterly in the Proceedings, and annual lists will be published in the index volumes of the Journal of the Chemical Society.

\section{Rhizomorph Formation in Armillaria mellea}

TEE importance of the rhizomorph in the evolution of fungi causing root disease has led to further experimental study of the inception and development of the rhizomorphs of a strain of the honey agaric (Armellaria mellea) under controlled cultural conditions (S. D. Garrett, Ann. Bot., N.S., 17, 65, 62 ; 1953). When a disk of mycelium taken from the margin of a colony is laid on a fresh agar substrate, mycelial growth begins within twenty-four hours and rhizomorph initials can be seen after seven days. The young rhizomorphs have a maximum growthrate which is about five times that of the normal hyphæ, the characteristic lobed appearance of colonies being largely due to this fact. An interaction was found between carbohydrate and nitrogen in their effects upon both the initial formation of rhizomorphs and their subsequent growth: as the carbohydrate concentration of the medium was increased, so the optimum concentration of nitrogen for the inception and growth of rhizomorphs increased. On a medium of suitable nutrient composition, no independent rhizomorph initials were observed apart from those originally formed around the inoculum disk. On the basis of experimental evidence it is considered that a certain threshold nutrient status is essential for rhizomorph initiation. Once a rhizomorph has started to grow on an agar plate, the depletion of nutrients in its vicinity prevents the formation of new initials.

\section{Microscopes and Accessory Equipment}

The new microscope catalogue of R. and J. Beck, Ltd. (69 Mortimer Street, London, W.1), covers a wide range of microscopes and accessories. It gives details of several new types including the reflecting microscope and the universal microscope, and apparatus is described for vertical phase-contrast illumination and fluorescence microscopy. A welcome addition for many workers will be the $35-\mathrm{mm}$. photomicrographic camera. A notable feature of the Beck equipment is that it is largely designed as interchangeable units. The catalogue has eighty pages, is pleasantly and clearly produced and should be of interest to all microscopists.

\section{Luminescence : Conference in Cambridge}

THE Electronics Group of the Institute of Physics is organizing a conference on luminescence, with particular reference to solid inorganic phosphors, which will be held in Cambridge during April 7-10, 1954. The first half-day of the meeting will include a lecture on the present scope of the subject and its developments since the last conference of this kind (Faraday Society, Oxford, 1938). The next day. April 8, will be devoted to papers on phosphor applications and future prospects, especially in the fields of cathode-ray tubes, lighting, electroluminescence and scintillation counting. Papers on the physical chemistry and atomic structure of phosphors, both sulphide and other types, and especially on the nature of the luminescence centre, will be read on April 9. On the last day more theoretical ideas on luminescence will be considered, such as the absorption, emission, transport and storage of energy, and the theory of activator systems. During the conference there will be an evening lecture with demonstrations on unsolved problems in luminescence. Further information can be obtained from Dr. S. 'T. Henderson, Institute of Physics, 47 Belgrave Square, London, S.W.1.

\section{British Institute of Management}

THE British Institute of Management has recently issued details of its pioneer executive development programme which provides for a series of four-weekly courses to be held at Management House, Hill Street, London, W.1, at intervals over the coming year. The first course opens on November 1. The programme is specially designed to meet the needs of the established executive in the $30-40$ age group whose experience to date has been in a particular specialization. The contents of the courses and methods employed are based on recognition of the fact that successful management depends substantially upon the possession of certain thought habits and attitudes of which analytical ability and a social sense are perhaps the most fundamental. Thus corporate life, syndicate work, exercises and reports, speech-training and committee work are vital ingredients of the programme-no wit less important than the informatory lectures which cover the broad field of management and the best current methods. Of particular interest to those concerned in the running of courses is the modification of the case-study procedure to a form appropriate to the maturity and experience of members of the course. In the American schools of business where the case-study method is a significant feature, the preoccupation is with students with little or no executive experience, and the task is therefore different. This facet of the programme is a recognition of a basic difference between contemporary provision in the American schools of business - -such as Harvard--where the majority of students have little or no previous experience, and the situation in Great Britain, where the climate of opinion demands that a man should have experience of industry before he undertakes a course of this kind which is designed to develop his managerial ability.

Another interesting feature is the concept of providing a course to aid the individual and personal development of the student. Thus the entire programme permits only twenty-four students working for the greater part of the time in smaller groups. In fact, the extent of personal development can be in direct proportion to the extent of individual participation. The lecturers and tutors are in the 